gesetzten kristalloiden Zustand scharf zu wnterscheiden und damit eine jetzt soviel bestrittene Frage aufzuklären.

\section{Die Grundlagen der Erregung und der Erregungsleitung in der lebendigen Substanz.}

Von Priv.-Doz. Dr. Walter Thörner, Bonn. I.

Mit der Frage nach den Grundlagen des Erregungsprozesses greifen wir eines der bedeu. tungsvollsten, aber verwickeltsten Probleme der allgemeinen Physiologie heraus. Alles, was wir an Lebensäuberungen 'der' Organismen wahrnehmen, alles ,Leben" überhaupt, ist im weitesten Sinne Erregung, unterhalten dureh die dauernd variierencen Einflüsse innerer und äuberer Faktoren, die wir die Lebensbedingungen nennen. Die Gesamtwirkung aller Lebensbedingungen ist eben das Leben. Da die Lebensbedingungen sich unaufhörlich ändern, so ist auch das ,Leben " kein stationärer Zustand, sondern ein kompliziert zusammengesetzter. Vorgang, der aus einer unabsehbaren Zahl ron Teilvorgängen besteht, die wir als Lebensprozesse bezeichnen, obwohl Leben erst das komplexe Ganze ist. Es ist demnach der Begriff absolutex Ruhe in einem lebendigen System (Ze'le, Organ, Organismus) niemals realisiert. Wir könnten von Ruhe nur sprechen, wenn wir unendlich kleine Zeiträume betrachteten, in denen die Anderungen der Lebensbedingungen unendlich klein wären. Darum hat die allgemeine Physiologie den Begriff der Ruhe relativ gefaßt, nämlich als einen Zustand des Gleichgewichts, in den sich die Lebensprozesse $z u$ den Änderungen der Lebensbedingungen setzen, wenn diese sich in gewissen Grenzen halten. Die lebendige Substanz paßt sich den jewei's herrschenden Bedingungen an.

Von diesem Zustand relativer Ruhe ausgehend bezeichnen wir als Erregung in engerem. Sinne jede Störung dieses Gleichgewichtszustandes, welche in einer Steigerung der Lebensprozesse besteht, als Lähmung dagegen eine solche im Sinne einer Herabsetzung der Lebensprozesse. Wir bezeichnen weiter als Reiz jede Anderung einor Lebensbedingung, dio zu einer Störung des Gleichgewichtes fuhrt, und müssen demnach erregende und lähmende Reize unterscheiden.

Damit ein ruhendes System aus seinem Gleichgewicht gebracht werde, müssen zwei Bedingungen erfüllt sein. Der Reiz, d. h. die Ändérung einer Lebensbedingung muB eine gewisse Größe erreichen und das lebendige System mul reizbar, d. h. erregbar, sein. Die Reizbarkeit oder Erregbarkeit ist eine allgemeine Eigenschaft aller lebendigen Substanz, in der eben nichts anderes zum Ausdruck kommt als die Abhängigkeit der Lebensprozesse, des 'Lebens überhaupt, von den Jebensbedingrungen. Wirkt ein Reiz längere Zeit oder dauerud ein, so setzt sich das lebendige System mit dieser veränderten Lebensbedingung ins Gleichgewicht; der Reiz wird dann zu einer newen Lebensbedingung, woraus wiederum erhellt, daB zwischen Reiz and Lebensbedingung. kein genereller, sondern nur ein gradueller Unterschied besteht. Die Reize werden dargestellt durch Intensitätsänderungen aller der Energieformen, deren Einflüssen auch die ruhende lebende Substanz unterworfen ist: strahlende Energie (Elektrizität, Licht, strahlende Wärme), mechanische Energie (Druck, Schwere, Schall), Wärme (Wärme und Kälte), chemische und osmotische Energie. Alle diese Energieformen kommen als Reize in Betracht, ihre Wirkung zeigt aber, so verschiedenartig die Reize sein mögen, nur graduelle Unterschiede, während die Art und Form der Reaktion, der Erregung, allein bestimmt wird durch Art and Zustand des gereizten lebendigen Systems. Bei einer bestimmten Form der lebendigen Substanz haben verschiedene Reize stets einen gleichartigen Reizerfolg, der die spezifische Reaktion des betreffenden Organs darstellt. Handelt es sich ja doch nur um eine Steigerung der in demselben schon in der Ruhe ablaufenden Lebensprozesse. Ein Muskel gibt immer eine Kontraktion, eine Sinneszelle des Auges immer eine Lichtempfindung, eine Drüsenzelle stets sekretorische Vorgänge, welcher Art auch die Reizang sei. Das ist das Gesetz von der spezifischen Erregbarkeit der lebendigen Substanz, das eine Erweiterung darstellt des schon von Johannes Müller aufgefundenen Gesetzes von der spezifischen Energie der Sinnesorgane.

Damit ist aber nicht gesagt, dab nun jede beliebige Reizform auf ein bestimmtes Organ den gleichen Grad der Wirksamkeit hätte. Das Gegenteil ist der Fall. Jedes lebende System spricht auf eine besondere Art von Reizen besonders leicht an, z. B. Nerv und Muskel auf elektrische Reize, die anscheinend dem physiologischen Reiz für diese Organe nahekommen. Tine wesentliche Rolle spielt hierbei sicherlich das Verhältnis zwischen der Geschwindigkeit des Reizablaufes und de Ablaufes der Lebensprozesse des gereizten Organes. Es mul da eine gewisse Übereinstimmung herrschen zur Errielung des günstigsten Reizerfolges. Formen mit langsamen Lebensprozessen reagieren leichter auf langsam verlaufende, solche mit schnellen dagegen besser auf schnell und steil verlaufende Reize. Viele Organe haben einen besonderen Apparat ausgebildet, um unter Fernhaltung aller übrigen nur eine Reizart, in reiner und verstärkter Form zur Wirkung kommen zu lassen, wie z. B. das Auge für Licht-, das Gehörorgan für Schallwellen, die Tastzellen für Druckreize usw. In solchen Fällen spricht man von adäquaten Reizen.

Die zweite Bedingung, neben der Erregbarkeit, für das Zustandekommen eines Reizerfolges liegt in der Größe des Reizes. Der Reiz muß eine gewisse Größe erreichen, die gegeben ist durch das 
Produkt aus Intensität und Dauer der Einwirkung, um die Reizschwelle des betreffenden lebendigen Systems zu überschreiten. Unter Reizschwelle versteht man die Reizgröbe, bei welcher eben ein erkennbarer Reizerfolg eintritt, während schwächere Reize ohne sichtbaren Erfolg bleiben. Der Begriff der Reizschwelle muß kurz nähex bestimmt werden, um einen Sinn zu erhalten. Die Reizschwelle hängt einerseits ab von der Art und Geschwindigkeit des Reizes, von dem Grad seiner Adäquatheit im Sinne des oben Gesagten, und andererseits von dem Erregbarkeitsgrade der betroffenen lebendigen Substanz, welcher sowohl bei demselben System, wie wir noch sehen werden, den verschiedensten Einflüssen unterliegt, als auch bei verschiedenen Formen lebendiger Substanz große Differenzen aufweist. Und drittens ist zu beachten, daB wir ja meist die Erregung gar nicht an dem von Reiz unmittelbar betroffe. nen Orte erkennen, sondern $d a B$ wir erst einen von dort fortgeleiteten und mehrfach übertragenen Reizerfolg an einem Erfolgsorgan, das uns als Indikator dient, zum Ausdruck kommen sehen. Wir bestimmen also eigentich für dieses die Reizschwelle, während vielleicht schon schwächere Reize genügen würden, um am Reizpunkte selbst Erregungen auszulösen.

Der äuBerlich wahrnehmbare Reizerfolg tritt niemals sofort im Augenblick der Reizung ein. Immer liegt eine wiederum spezifisch sehr verschieden lange Zeit zwischen dem Moment des Reizes und dem Beginn der sichtbaren Reaktion, z. B. zwischen dem Augenbiick der Reizung eines Muskels oder seines Nerven und der Zuckung des Muskels. Man nennt diese Zeit die Latenzzent, das Stadium der verborgenen Erregung. Thre Daner hängt $a b$. von der Länge des Weges, den die Erregung vom Reizpunkte bis zum Erfolgsorgan zu durchlaufen hat; man findet daher in der Vergleichung der Latenzzeiten für verschieden lange Strecken ein Mittel zur Bestimmung der Fortleitungsgeschwindigkeit der Erregung. Die Dauer des Latenzstadiums ist ferner abhängig von dem.Zustand der lebendigen Substanz, von der spezifischen Art und Geschwindigkeit der Lebensprozesse in ihr, durch welche die Geschwindigkeit des Erregungsablaufes bedingt wird. Davon werden wir noch zu sprechen haben.

Die Vorgänge, die sich in der Latenzzeit verbergen, können wir ganz allgemein in drei Fo'gezustände der Reizung gliedern, je nach dem. Ort, an dem wir sie betrachten. Die am Reizpunkte selbst durch den Reiz ausgelöste Gleichgewichtsstörung besteht in Veränderungen, die wir als primären Reizerfolg oder primäre Erregung bezeichnen. Diese geben Veranlassung zu weiteren Störungen in den benachbarten Teilen, die sich über das ganze lebendige System räumlich ausbreiten, das ist die Erregungsleitung oder der sekundäre Reizerfo.g. Und schlieBlich kann ein Arfolgsorgan in äuBerlich sichtbare Erregung geraten, das uns mit dem tertiären Reizerfolg den
Ablauf des ganzen Prozesses anzeigt. Dabei kann sich der gesamte Reizworgang sowohl auf ein einzelnes lebendiges System beschränken, wie z. B. bei direkter Muskelreizung. wie auch über mehrere Systeme nacheinander ablaufen, in dem das eine System das andere in Erregung versetzt, wie wir es z. B. von der Reizung eines Nervmuskelpräparates oder von den Rückenmarksreflexen kennen und wie es bei den physiologischen Reizvorgängen wohl meist verwirkicht ist.

Die den einzelnen Reizerfolgen zugrunde liegenden Prozesse brauchen sich in ihrem speziellen Ablauf nicht $z u$ gleichen. Ihnen allen gemeinsam aber ist, daß sie Auslösungsvorgänge darstellen, die, durch den Reiz einmal in Gang gebracht, weiterhin unabhängig von ihm automatisch ablaufen. Sie alle sind Erregungen, deren Grundprinzipien wir im folgenden darzulegen versuchen wollen.

Wir betrachten dabei zweckmäBig das Leben unter der Form des Stoffwechsels, den wir als grundlegenden unter allen Lebensvorgängen ansehen dürfen. Unausgesetzt zerfal'en grobe komplexe Moleküle, die die lebendige Substanz zusammensetzen, zu kleineren Bruchstücken, und unausgesetzt werden aus frischen Materialien neve Molekule lebendiger Substanz wieder aufgebaut. Es besteht demnach dieser Stoffumsatz aus zwei Phasen, die nach dem Prinzip der Selbststeverung miteinander weehseln: Dissimilation und Assimilation, Zerfall und Aufbau. Die Dissimilation ein schnell verlaufender AufspaltungsprozeB großer Moleküle, die Assimilation sich unmittelbar anschließend, aber langsamer vollendend, in we.cher unter Heranführung neuer Nahrungsstoffe und unter Beseitigung der den weiteren Zerfall hemmenden Zerfallsprodukte die lebendige Substanz zum status quo ante wieder aufgebaut wird. An diesen Stoffumsatz ist unlösbar ein Energieumsatz gebunden, wie ja bei allen chemischen Vorgängen Energie entweder verbraucht oder frei wird. Da beim Zerfall der großen sehr locker gebundenen Moleküle zu k’einen Bruchstücken Moleküle mit festeren Bindungen entstehen, also schwache Affinitäten gelöst werden und starke sich binden, so geht die Dissimilation mit Entfaltung freier aktueller Energie einher, die an den lebendigen Systemen als Wäme, Bewegung, Licht, Fektrizität usw. zum Ausdruck kommt und deren Gesamterscheinungsformen wir als Lebensäußerungen zu bezeichnen pflegen. Umgekehrt vollzieht sich der Wiederauf. bau never komplexer Noleküle, die Assimilation nnter Verbrauch von Energie, die dem lebendigen System als chemische Energie in Gestalt von Nahrung, Sauerstoff, Kohlensäure, ferner als Licht und Wärme zufießt.

Unterhalten wird dieser Stoffwechsel durch die Reize der danernd sich ändernden Lebensbedingungen. Jedoch besteht in den ,ruhenden Systemen ein Gleichgewicht, indem in der Zeit- 
einheit ebensoviel lebendige Substanz wieder aufgebaut wie zersetzt wird. Dies Gleichgewicht bleibt erhalten, solange die Schwankungen der Lebensbedingungen eine gewisse Grenze nicht überschreiten. Wir sprechen von Ruhestoffwechsel, wenngleich, wie schon erwähnt, absolute Ruhe nirgends in der Welt der Organismen reajisiert ist und einem Stillstand des Stoffwechsels gleich käme, welcher Tod bedeuten würde.

Diesem durch sein Gleichgewicht charakterisierten Ruhestoffwechsel stellen wir den Reizoder Erregungsstoffwechsel, auch Funktionsstoffwechsel gegenüber. Wirkt ein Reiz auf die lebendige Substanz ein in Gestalt einer gröberen Änderung einer Lebensbedingung, so wird das Stoffwechselgleichgewicht gestört. Es erfo.gt eine Geschwindigkeitsänderung im Ablauf seiner einzelnen Phasen. Handelt es sich um eine Beschleunigung, so nenuen wir den Reizerfolg Erregung und den Reiz einen erregenden, während wir bei Verlangsamung von Lähmung und. Tähmendem Reiz sprechen. Da aber der Stoffumsatz aus zwei entgegengesetzt verlaufenden Phasen besteht, so kann die Reizwirkung primär an jeder einzelnen angreifen, d. h. es kann zu einer primären Beschleunigung bezw. Verlangsamung entweder der dissimilatorisehen oder der assimilatorischen Vorgänge kommen, und weiterhin können wir uns vorstellen, daß beide Stoffwechselphasen gleichzeitig beeinflußt werden, entweder in gleichem oder in entgegengesetztem Sinne. Es sind demnach mehrere Möglichkeiten der Reizwirkung gegeben. Praktisch verringert sich für uns die Zahl der Fälle. Da uns nur der Vorgang der Erregung hier interessiert, wollen wir von den primär tähmenden Reizen ganz absehen, wie sie hauptsächlich von der Gruppe narkotisch wirkender Substanzen dargestellt werden.

Für die erregenden Reize ist nun bedeutsam, dab wir außer dem Reiz der Nahrungszufuhr mit Sicherheit keinen anderen kennen, welcher primär die Assimilationsprozesse steigert. Vielmehr greifen die erregenden Reize im allgemeinen an der Dissimilationsphase an, indem sie einen beschleunigten Zerfall lebendiger Substanz auslösen. Dem Zerfall folgt dann aber unmittelbar durch Selbststeuerang der assimilatorische Neuaufbau, die Wiederherstellung zerfallsbereiter Massen. Utberschreitet aber ein primär erregender Reiz ein gewisses $\mathrm{MaB}$ an Dauer oder Intensität, so schlägt die Erregung in Lähmung um, indem dann die nach dem Zerfall einsetzenden Aufbauprozesse in der Zeiteinheit nicht soviel neuer Substanz wiederherstellen können, als durch die Reizung zum Zerfall gebracht wird. Beispiele hierfür finden wir in der Ermüdung durch rhythmische Reizung und in dem Utbergang von Wärmeerregung in Wärmelähmung, wovon noch zu sprechen sein wird. Dieser Umschlag in lähmende Wirkung ist allen primär erregenden Reizen, welche gewisse Grenzen überschreiten, eigentümlich, eine Tatsache, die schon A.v. Humboldt bekannt war, der ror 140 Jahren schrieb: ,Wärme im Übermaß angewandt bringt, wie jede reizende Potenz, Schwäche hervor."

Unterziehen wir nun die bei der Erregung sich abspielenden Vorgänge einer näheren Betrachtung, so tritt uns als erste die Frage nach der Art des chemischen Geschehens bei der Erregung entgegen." Als Endprodukt des Erregungsstoffwechsels der aëroben Organismen erscheinen in den Ausscheidungen überwiegend Oxyde, wie Wasser, Kohiensäure und andere Säuren, also relativ einfache chemische Körper in Gestalt kleiner festgefügter Moleküle. Da wir andererseits wissen, daß die lebendige Substanz aus sehr komplexen und hochkompliziert gebauten chemischen Körpern zusammengesetzt ist, neben Fetten und Kohlehydraten und anderem vor allem aus Eiweißmolekülen, die an ihrem Kern die verschiedensten Atomgruppen in lockeren Bindungen tragen mögen, so müssen wir annehmen, daß diese großen Moleküle es sind, an denen sich der Erregungsstoffwechsel abspielt. Sie zerfallen in der Dissimilationsphase desselben und liefern unter Einwirkung dés Sauerstoffs jene als Oxyde erscheinenden Endprodukte. Gerade aus diesen letzteren aber können wir weiter schließen, daß im Erregungsstoffwechsel der Aërobier ${ }^{1}$ ) im wesentlichen nur die stickstoffrreien Atomgruppen beteiligt sind, nicht der Eiweißkern. Wenigstens sehen wir in den Ausscheidungen bei Arbeitsleistung stets nur eine Vermehrung der stickstofffreien oxydierten Endprodukte, aber keine vermehrte Stickstoffausscheidung. Demgegenüber ist im Ruhestoffwechsel wohl die ganze lebendige Substanz, auch das Eiweiß, exgriffen. Darin besteht ein gewisser qualitativer Unterschied zwischen dem Reiz- oder Funktionsstoffwechsel und den Umsetzungen in der Ruhe. Der Erregungsstoffwechsel beruht eben auf einer Steigerung nicht aller, sondern nur gewisser Teilprozesse des Ruhestoffwechsels, der mit seinen übrigen Gliedern ungestört weiter abläuft.

Wir haben es also im Erregungsstoffwechsel mit einer oxydativen Aufspaltung, einer Verbrennung zu tun. Man kann den Vorgang speziell der Dissimilation gut mit einer Explosion vergeichen, bei welcher auch ein sehr schnell verlaufender oxydativer Zerfall ausgelöst wird. Da. bei bleibt es allerdings unentschieden, ob beim Zerfall in der lebendigen Substanz schon gleich die ersten Phasen oxydativ verlaufen, oder ob anfangs eine einfache anoxydative Spatung der Moleküle erfolgt und erst nachträglich die gröberen, unvollkommenen Spaltprodukte weiter verbrannt werden. DaB anoxydative Spaltungen im Erregungsstoffwechsel möglich sind, beweisen die anaëroben Organismen, welche ohne Sauerstoff leben können oder müssen. Andererseits spricht, für einen von vornherein oxydativen Zerfall bei den aëroben Lebewesen die daraus resultierende

1) Wesen, für die freier Sauerstoff unentbebrlich ist. 
viel gröBere Energieproduktion. Jedenfalls spielt bei den Aërobiern der Sauerstoff eine gewaltige Rolle. Alle ihre Organo stelien ihren Erregungsstoffwechsel, später auch ihren Ruhestoffwechsel ein, wenn ihnen Sauerstoff fehlt, sie werden unerregbar und sterben ab.

Auch bezüglich der Energieentfaltung trifft der Vergleich mit Explosionsvorgängen zu. Wie bei diesen werden in der Zerfallsphase des Erregungsstoffwechsels infolge der Absättigung starker Affinitäten, speziell des Sauerstoffs, grobe Energiemengen frei. Chemische Energie wird umgewandelt in andere Energieformen, an denen wir äuberlich die Erregung erkennen. Sie treten auf, um nur einige zu nennen, als Bewegung, z. B. bei der Muskelkontraktion, als Wärme, die wir messen, als Elektrizität, die wir in Gestalt der Aktionsströme von erregten lebendigen Systemen ableiten können. An der Größe der Energieentfaltung haben wir ein Maß für die Größe der Erregung.

Nun ist aber mit dem Ablauf des Zerfalls der Erregungsprozeb keineswegs beendet. Hier läBt uns der Vergleich mit der Explosion im Stich. Schon während des Zerfalls tritt die Selbststeuerung des Stoffwechsels in Kraft, die der lebendigen Substanz eigentümlich ist. Es beginnt die Assimilation, die dann nach dem Aufhören der Dissimilation allein das Feld hat. Thr Verlauf in der Zeit ist weniger schnell, anfangs steiler, dann immer langsamer, je näher sie der Vollendung kommt. Unter Assimilation fassen wir die Gesamtheit der Prozesse zusammen, die zur vollen Restitution der lebendigen Substanz führen. Es kommt da einerseits in Betracht der Wiederaufbau neuer zerfallsfähiger Molekitle aus neuen Nährmaterialien und vielleicht unter Einfügung von Sauerstoff, andererseits die auf weiterer Oxydation und auf Diffusion beruhende Beseitigung der Produkte der Zerfallsreaktionen, deren Anhäufung gemäß dem Massenwirkungsgesetz den weiteren Ablauf der Umsetzungen hemmen und zum Stillstand bringen würde.

Als Grundlage des Erregungsvorganges haben wir somit eine zweiphasische Stoffwechselschwankung anzunehmen, die Dissimilation, dis durch den Reiz ausgelost wird, und die Assimilation, die den status quo ante wiederherstellt. Wegen des zweiphasischen Charakters ides Erregungsstoffwechsels spricht man auch von Erregungswelle. Die unmittelbare Folge dieses Stoffwechsels ist ein Energiewechsel, der uns in bestimmten Lebenserscheinungen die Erregung zum Ausdruck bringt.

Wir haben nun den Erregungsprozeß weiter in zweierlei Finsicht zu betrachten: einmal seinen Ablauf in der Zeit, zweitens seinen Ablauf im Raum.

Der Ablauf in der Zeit ist gleichbedeutend mit der Geschwindigkeit, mit welcher sich die beiden Stoffwechselphasen, dio Dissimilation und die Assimilation, vollziehen. Die Dauer der Erregung, die wir vom Beginn des Zerfalls bis zur vollende- ten Restitution rechnen, ist bei verschiedenen Formen lebendiger Substanz sehr verschieden lang, sie hängt eben ab von der Geschwindigkeit der spezifischen chemischen Umsetzungen, die wiederum eine Funktion der Art der reagierenden chemischen Körper ist.

Die Gesamtdauer einer Erregung beträgt am Nerven des Frosches bei $18^{\circ} \mathrm{C}$ etwa 0,1 Sekunde. Sie ist bei fast al'en anderen lebendigen Systemen wesentlich länger. Wie kommen wir zu solchen Zahlen, wie können wir die Schwingungsdauer einer Erregungswelle bestimmen?

Da hilft uns die sehr wichtige Erkenntnis, dab während des Bestehens einer Erregung die Erregbarkeit des betreffenden Systems für einen neuen Reiz beeinflußt ist, und zwar herabgesetzt oder gänzlich aufgehoben. Wir können daher eine Er. regung, gesetzt durch einen ersten Reiz, erst dann als vollständig abgeklungen ansehen, wenn ein folgender zweiter Reiz wieder normal wirksam ist, wieder eine maximale Erregung auslöst. Lassen wir die Reize schneller aufeinander folgen, so werden die Erregungen immer kleiner, und wir erhalten die noch zu besprechenden Erscheinungen der Ermüdung. Demnach entsprechen die zeitlichen Abstände der Reize, bei denen eben keine Ermüdung mehr eintritt, der Dauer der einzelnen Erregung.

\section{Besprechungen.}

Müller, Alois, Die Referenzflächen des Fimmels und der Gestirne. Die Wissenschaft Bd. 62. Braunschweig, Fr. Vieweg u. Sohn, 1918. VIII, 162 S. und $20 \mathrm{Ab}$ bild. Preis geh. M. 5,60, geb. M. 7,60.

Die Theorien des Raumsehens kann man prinzipiell in synthetische-erklarende and in analytische-beschreibende scheiden; jene wollen die Raumvorstellungen. aus einer Synthese ursprünglich gegebener, d. h. ale solche hypothetisch angenommener einfachster Empfindungen hervorgehen laseen, diese verstehen sie als Produkte einer Differenzierung der ursprünglich gegebenen sinnlichen Wahrnehmungsinhalte in ihren Beziehungen zu den Reizen. Als wiehtigstes Problem behandelt jede Theorie des Raumsehens das Verbältnis des Sehraumes zum wirklichen Raume bezw. das Ver. bältnis des Vorstellungsraumes zu dem begrifflichen objektiven Raume. Die synthetischen Theorien suchen entsprechend threr allgemeinen erkenntristheoretischen bezw. metaphysischen Orientierung bezüglich der $\mathrm{Na}$ tur des "Gegebenen" und bezüglich des Wesens der Er. kenntnis und ibres Zieles diese Probleme entweder aui dogmatisch-realietischer oder auf idealistischer Grundlage zu lösen. Im ersteren Falle sind entweder der objektive Raum selbst oder räumliche Verhältnisise als Realitäten gegeben. Der Wahrnehmungsprozeß ist dann im Prinzip ein Abbildungsprozeß, die Erscheinungen haben eine besondere Realität im Bewubtsein und ihrem Unsprung gemäß ihre besonderen Strukturgesetze. Im qweiten Falle sind die Raumvorstellungen besondere Akte des..Subjekts, sogenannte Objektivationen oder Projektionen; ihr Zusammenhang ist ein gesetzmäBig konstruktiver. In beiden Fällen handelt es aich m. E. um Theoriebildungen, die den beobachtbaren Tatsachen nicht gerecht werden können. 